\title{
Strong meager properties for filters
}

\author{
by
}

Claude L a fl a m me (Calgary, Alta.)

\begin{abstract}
We analyze several "strong meager" properties for filters on the natural numbers between the classical Baire property and a filter being $\mathrm{F}_{\sigma}$. Two such properties have been studied by Talagrand and a few more combinatorial ones are investigated. In particular, we define the notion of a $\mathrm{P}^{+}$-filter, a generalization of the traditional concept of P-filter, and prove the existence of a non-meager $\mathrm{P}^{+}$-filter. Our motivation lies in understanding the structure of filters generated by complements of members of a maximal almost disjoint family.
\end{abstract}

1. Introduction. We investigate several "smallness" properties of filters as strengthening of the meager property. Indeed, although a meager filter might be thought of as small, it may happen for example that adding one set to a meager filter yields an ultrafilter (see the section on examples). Our motivation lies in the unresolved relationship between the cardinal $\mathfrak{a}$, the minimum size of a maximal almost disjoint (mad) family, and $\mathfrak{d}$, the minimum size of a dominating family. The similar question relating $\mathfrak{a}$ with $\mathfrak{b}$, the minimum size of an unbounded family, has been settled by Shelah as independent from ZFC in [4]. Part of the problem lies in the technical question whether filters generated by the complement of members of a mad family can be diagonalized in a forcing extension without adding an unbounded real. This loosely means that we must understand the different possibilities for a filter to code an unbounded function when diagonalized. Partial results were obtained in [3] where it became apparent that strong meager properties are required on filters for this program to succeed. It is the goal of this paper to analyze some of these stronger meager properties.

Our terminology is standard but we review the main concepts and notation. The natural numbers will be denoted by $\omega,{ }^{\omega} 2$ and ${ }^{\omega} \omega$ denote the

1991 Mathematics Subject Classification: Primary 04A20; Secondary 03E05, 03E15, $03 \mathrm{E} 35$.

Key words and phrases: filter, meager, Baire property.

This research was partially supported by NSERC of Canada. 
collection of functions from $\omega$ to 2 and to $\omega$ respectively; similarly, $\wp(\omega)$ and $[\omega]^{\omega}$ denote the collections of all subsets and all infinite subsets respectively. Two orderings are standard, the first one for subsets of $\omega$ is $A \subseteq^{*} B$ if $A \backslash B$ is finite, the second one for functions is $f \leq^{*} g$ if $f(n) \leq g(n)$ for all but finitely many $n$. We can view members of $\wp(\omega)$ as members of ${ }^{\omega} 2$ by considering their characteristic functions. We equip ${ }^{\omega} 2$ with the product topology of the discrete space $\{0,1\}$. A basic neighbourhood is then given by sets of the form

$$
\mathcal{O}_{s}=\left\{f \in \omega^{\omega} 2: s \subseteq f\right\}
$$

where $s \in{ }^{<\omega} 2$, the collection of finite binary sequences. The terms "nowhere dense", "meager", "Baire property" all refer to this topology.

A filter is a collection of subsets of $\omega$ closed under finite intersections, supersets and containing all cofinite sets; it is called proper if it contains only infinite sets. Given a collection $\mathcal{X} \subseteq \wp(\omega)$, we let $\langle\mathcal{X}\rangle$ denote the filter generated by $\mathcal{X}$. For a filter $\mathcal{F}, \mathcal{F}^{+}$denotes the collection of all sets $X$ such that $\langle\mathcal{F}, X\rangle$ is a proper filter; it is useful to notice that $X \in \mathcal{F}^{+}$if and only if $X^{\mathrm{c}} \notin \mathcal{F}$. We say that $X \in[\omega]^{\omega}$ diagonalizes a filter $\mathcal{F}$ if $X \subseteq^{*} Y$ for all $Y \in \mathcal{F}$. The Fréchet filter is the collection of cofinite sets, denoted by Fr. Given two filters $\mathcal{F}$ and $\mathcal{G}$, we form a third filter by

$$
\mathcal{F} \otimes \mathcal{G}=\{X \subseteq \omega \times \omega:\{n:\{m:(n, m) \in X\} \in \mathcal{G}\} \in \mathcal{F}\} .
$$

This filter can be viewed as a filter on $\omega$ if desired by fixing a bijection between $\omega \times \omega$ and $\omega$.

The following important result characterizes meager filters in terms of combinatorial properties.

Proposition 1.1 (Talagrand [6]). The following are equivalent for a filter $\mathcal{F}$ :

1. $\mathcal{F}$ has the Baire property.

2. $\mathcal{F}$ is meager.

3. There is a sequence $n_{0}<n_{1}<\ldots$ such that

$$
(\forall X \in \mathcal{F})\left(\forall^{\infty} k\right) X \cap\left[n_{k}, n_{k+1}\right) \neq \emptyset .
$$

A family $\mathcal{F} \subseteq{ }^{\omega} \omega$ is called bounded if

$$
\left(\exists g \in{ }^{\omega} \omega\right)(\forall f \in \mathcal{F}) f \leq^{*} g .
$$

Similarly, a family $\mathcal{F} \subseteq{ }^{\omega} \omega$ is called dominating if

$$
\left(\forall g \in{ }^{\omega} \omega\right)(\exists f \in \mathcal{F}) g \leq^{*} f .
$$

The cardinals $\mathfrak{b}$ and $\mathfrak{d}$ denote respectively the minimum cardinality of an unbounded or dominating family. 


\section{Strong meager properties}

Definition 2.1. Let $\mathcal{F}$ be a filter on $\omega$.

1. $\mathcal{F}$ is called completely meager (Talagrand [7]) if the filter $\langle\mathcal{F}, X\rangle$ is meager whenever $X^{\mathrm{c}} \notin \mathcal{F}$.

2. $\mathcal{F}$ is called hereditarily meager if for each $f \in{ }^{\omega} \omega$, the filter $f(\mathcal{F})$ is meager (or improper).

3. $\mathcal{F}$ is called weakly hereditarily meager if for each $f \in{ }^{\omega} \omega$, either $f^{-1}(i)$ is compatible with $\mathcal{F}$ for some $i$, or else $f(\mathcal{F})$ is meager.

4. $\mathcal{F}$ has the strong Baire property (Talagrand [7]) if $\mathcal{F} \cap \mathcal{C}$ has the Baire property relative to $\mathcal{C}$ for each closed $\mathcal{C} \subseteq \wp(\omega)$.

5. $\mathcal{F}$ is a $\mathrm{P}^{+}$-filter if given any decreasing sequence $\left\langle X_{0}{ }^{*} \supseteq X_{1}{ }^{*} \supseteq X_{2}{ }^{*}\right.$ $\supseteq \ldots\rangle$ from $\mathcal{F}^{+}$, there is an $X \in \mathcal{F}^{+}$such that $X \subseteq^{*} X_{n}$ for each $n$.

6. $\mathcal{F}$ is a strong $\mathrm{P}^{+}$-filter if given any sequence $\left\langle X_{0}, X_{1}, X_{2}, \ldots\right\rangle$ from $\mathcal{F}^{+}$, there is an $f \in{ }^{\omega} \omega$ such that for all $X \in \mathcal{F}, X \cap X_{n} \cap f(n) \neq \emptyset$ for all but finitely many $n$.

We shall prove the following implications between these notions. In a subsequent section, we shall show by examples that many of these implications do not reverse. Some open questions remain.

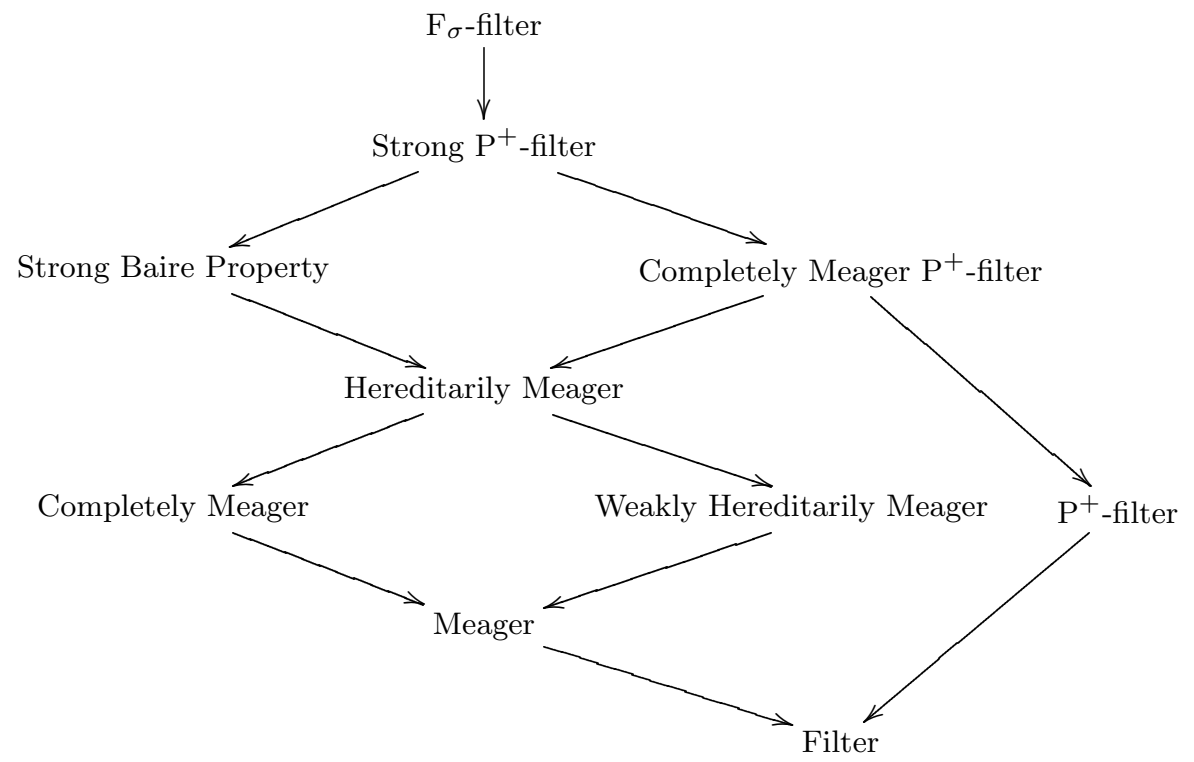

The following lemmas will only reformulate these definitions into more uniform and manageable ones; the proofs of the first three are left to the reader.

LEMMA 2.2. The following are equivalent for a given filter $\mathcal{F}$ :

1. $\mathcal{F}$ is weakly hereditarily meager. 
2. For each sequence $\left\langle X_{i}: i \in \omega\right\rangle$ such that $\bigcup_{i} X_{i} \in \mathcal{F}$ and each $X_{i}^{\mathrm{c}} \in \mathcal{F}$,

$$
\left(\exists n_{0}<n_{1}<\ldots\right)(\forall Y \in \mathcal{F})\left(\forall^{\infty} k\right) Y \cap \bigcup_{i \in\left[n_{k}, n_{k+1}\right)} X_{i} \neq \emptyset .
$$

LEMMA 2.3. The following are equivalent for a given filter $\mathcal{F}$ :

1. $\mathcal{F}$ is completely meager.

2. For each sequence of finite sets $\left\langle X_{i}: i \in \omega\right\rangle$ such that $\bigcup_{i} X_{i} \in \mathcal{F}^{+}$,

$$
\left(\exists n_{0}<n_{1}<\ldots\right)(\forall Y \in \mathcal{F})\left(\forall^{\infty} k\right) Y \cap \bigcup_{i \in\left[n_{k}, n_{k+1}\right)} X_{i} \neq \emptyset .
$$

LEMMA 2.4. The following are equivalent for a given filter $\mathcal{F}$ :

1. $\mathcal{F}$ is hereditarily meager.

2. For each sequence $\left\langle X_{i}: i \in \omega\right\rangle$ such that $\bigcup_{i} X_{i} \in \mathcal{F}^{+}$and each $X_{i}^{\mathrm{c}} \in \mathcal{F}$,

$$
\left(\exists n_{0}<n_{1}<\ldots\right)(\forall Y \in \mathcal{F})\left(\forall^{\infty} k\right) Y \cap \bigcup_{i \in\left[n_{k}, n_{k+1}\right)} X_{i} \neq \emptyset .
$$

3. For each sequence $\left\langle X_{i}: i \in \omega\right\rangle$ such that $\bigcup_{i} X_{i} \in \mathcal{F}, \bigcup_{i<k} X_{i} \notin \mathcal{F}$ for each $k$ and only finitely many $X_{i}$ belong to $\mathcal{F}^{+}$,

$$
\left(\exists n_{0}<n_{1}<\ldots\right)(\forall Y \in \mathcal{F})\left(\forall^{\infty} k\right) Y \cap \bigcup_{i \in\left[n_{k}, n_{k+1}\right)} X_{i} \neq \emptyset .
$$

LEMma 2.5. The following are equivalent for a given filter $\mathcal{F}$ :

1. $\mathcal{F}$ is a completely meager $\mathrm{P}^{+}$-filter.

2. For each sequence $\left\langle X_{i}: i \in \omega\right\rangle$ such that $\bigcup_{i \geq n} X_{i} \in \mathcal{F}^{+}$for each $n$,

$$
\left(\exists h \in{ }^{\omega} \omega\right)\left(\exists n_{0}<n_{1}<\ldots\right)(\forall Y \in \mathcal{F})\left(\forall^{\infty} k\right) Y \cap \bigcup_{i \in\left[n_{k}, n_{k+1}\right)} X_{i} \cap h(i) \neq \emptyset .
$$

3. For each sequence $\left\langle X_{i}: i \in \omega\right\rangle \subseteq \mathcal{F}^{+}$,

$$
\left(\exists h \in{ }^{\omega} \omega\right)\left(\exists n_{0}<n_{1}<\ldots\right)(\forall Y \in \mathcal{F})\left(\forall^{\infty} k\right) Y \cap \bigcup_{i \in\left[n_{k}, n_{k+1}\right)} X_{i} \cap h(i) \neq \emptyset .
$$

Proof. $1 \Rightarrow 2$. Let $\mathcal{F}$ be a completely meager $\mathrm{P}^{+}$-filter and fix a sequence $\left\langle X_{i}: i \in \omega\right\rangle$ such that $\bigcup_{i>n} X_{i} \in \mathcal{F}^{+}$for each $n$.

Define $Y_{n}=\bigcup_{i \geq n} X_{i}$. Then $Y_{0} \supseteq Y_{1} \supseteq \ldots$ is a decreasing sequence from $\mathcal{F}^{+}$and therefore there is an $X \in \mathcal{F}^{+}$almost included in each $Y_{i}$. Since $\langle\mathcal{F}, X\rangle$ must be meager, choose $m_{0}<m_{1}<\ldots$ such that

$$
(\forall Y \in \mathcal{F})\left(\forall^{\infty} k\right) Y \cap X \cap\left[m_{k}, m_{k+1}\right) \neq \emptyset .
$$

Now choose $n_{0}<n_{1}<\ldots$ and $h \in{ }^{\omega} \omega$ such that

$$
(\forall k)(\exists l)\left[m_{l}, m_{l+1}\right) \cap X \subseteq \bigcup_{i \in\left[n_{k}, n_{k+1}\right)} X_{i} \cap h(i) .
$$


$2 \Rightarrow 3$ is immediate.

$3 \Rightarrow 1$. To show that $\mathcal{F}$ is a $\mathrm{P}^{+}$-filter, fix a decreasing sequence $X_{0} \supseteq$ $X_{1} \supseteq \ldots$ from $\mathcal{F}^{+}$. By assumption, there are $h \in{ }^{\omega} \omega$ and a sequence $n_{0}<$ $n_{1}<\ldots$ such that

$$
(\forall Y \in \mathcal{F}) Y \cap \bigcup_{i \in\left[n_{k}, n_{k+1}\right)} X_{i} \cap h(i) \neq \emptyset .
$$

If we let $X=\bigcup_{k} X_{n_{k}} \cap h\left(n_{k}\right)=\bigcup_{i} X_{i} \cap h(i)$, then $X \in \mathcal{F}^{+}$and is almost included in each $X_{i}$.

To show that $\mathcal{F}$ is completely meager, let $X \in \mathcal{F}^{+}$be given and use the assumptions on $X_{i}=X \backslash i$.

Now we are ready to prove the diagram implications; in view of the above lemmas, all are straightforward except possibly two of them which we prove in detail.

Proposition 2.6. A strong $\mathrm{P}^{+}$-filter has the strong Baire property.

Proof. We actually show that a strong $\mathrm{P}^{+}$-filter has the Baire property relative to any set $\mathcal{X} \subseteq{ }^{\omega_{2}}$. Indeed, let

$$
\mathcal{T}=\left\{s \in{ }^{<\omega_{2}}: \mathcal{O}_{s} \cap \mathcal{X} \subseteq \mathcal{F}\right\},
$$

and put $\mathcal{O}=\bigcup_{s \in \mathcal{T}} \mathcal{O}_{s}$. We show that $(\mathcal{F} \cap \mathcal{X}) \triangle(\mathcal{O} \cap \mathcal{X})$ is meager in $\mathcal{X}$.

For this, let $\mathcal{S}=\left\{s \in<^{<} 2: \mathcal{O}_{s} \cap \mathcal{X} \neq \emptyset\right.$ and $\left.\mathcal{O}_{s} \cap \mathcal{X} \nsubseteq \mathcal{F}\right\}$; enumerate $\mathcal{S}$ as $\left\langle s_{n}: n \in \omega\right\rangle$. For each $n$, choose $A_{n} \in\left(\mathcal{O}_{s_{n}} \cap \mathcal{X}\right) \backslash \mathcal{F}$ and put $X_{n}=$ $A_{n}^{\mathrm{c}} \backslash \operatorname{lh}(s) \in \mathcal{F}^{+}$. By assumption, there is an $h \in{ }^{\omega} \omega$ such that for all $Y \in \mathcal{F}$, $Y \cap X_{n} \cap h(n) \neq \emptyset$ for all but finitely many $n$. Define now

$$
\mathcal{M}_{k}=\left\{X:(\forall l \geq k) X \cap X_{l} \neq \emptyset\right\} \backslash \mathcal{O} .
$$

Clearly $(\mathcal{F} \backslash \mathcal{O}) \subseteq \bigcup_{k} \mathcal{M}_{k}$ and each $\mathcal{M}_{k}$ is nowhere dense in $\mathcal{X}$.

Proposition 2.7. Every $\mathrm{F}_{\sigma}$-filter is a strong $\mathrm{P}^{+}$-filter.

Proof. Let $\mathcal{F}=\bigcup_{n} \mathcal{C}_{n}$ be a countable increasing union of closed sets $\mathcal{C}_{n} \subseteq[\omega]^{\omega}$ and $\left\langle X_{n}: n \in \omega\right\rangle \subseteq \mathcal{F}^{+}$. Put $\mathcal{C}=\left\{X \cup n:(\exists n) X \in \mathcal{C}_{n}\right\}$ and define $\mathcal{C}_{n}^{\prime}=\mathcal{C} \cup X_{n}$. Choose $h \in{ }^{\omega} \omega$ such that for all $n$,

$$
\left(\forall Y, Z \in \mathcal{C}_{n}^{\prime}\right) Y \cap Z \cap[n, h(n)) \neq \emptyset .
$$

The existence of such a function is guaranteed by the fact that $\mathcal{C}_{n}^{\prime}$ is closed. Now if $Y \in \mathcal{F}$, say $Y \in \mathcal{C}_{i}$, then $Y \cup i \in \mathcal{C}$ and therefore $(Y \cup i) \cap X_{n} \cap h(n) \neq \emptyset$ for all $n$, and thus $Y \cap X_{n} \cap h(n) \neq \emptyset$ for all $n \geq i$ as desired.

3. Examples. We now produce examples showing that many implications in the diagram are not reversible. 
EXAMPLE 3.1 (A weakly hereditarily meager filter which is not completely meager). Let $\mathcal{U}$ be any ultrafilter not containing say the even numbers $E$. Define $\mathcal{F}=\langle\{E \cup X: X \in \mathcal{U}\}\rangle$. Then $\left\langle\mathcal{F}, E^{\mathrm{c}}\right\rangle=\mathcal{U}$ is not meager and thus $\mathcal{F}$ is not completely meager.

Now let $\left\langle X_{i}: i \in \omega\right\rangle$ be given such that $\bigcup_{i} X_{i} \in \mathcal{F}$ and each $X_{i}^{\mathrm{c}} \in$ $\mathcal{F}$. Choose a sequence $n_{0}<n_{1}<\ldots$ such that for each $k,(E \backslash k) \cap$ $\bigcup_{i \in\left[n_{k}, n_{k+1}\right)} X_{i} \neq \emptyset$. This is possible as $E \cap \bigcup_{i<k} X_{i}$ is finite for each $k$. Since any $Y \in \mathcal{F}$ almost contains $E$, we conclude that $\mathcal{F}$ is weakly hereditarily meager by Lemma 2.2 .

EXAMPLE 3.2 (A completely meager filter which is not weakly hereditarily meager). This example comes essentially from [7], where a completely meager filter without the strong Baire property is produced. Let again $\mathcal{U}$ be an ultrafilter and let $\mathcal{F}=\mathcal{U} \otimes F r$.

Claim 1. $\mathcal{F}$ is completely meager.

Indeed, let $X \subseteq \omega \times \omega$ be compatible with $\mathcal{F}$; this means that $X_{n}=$ $\{(n, m): m \in X\}$ is infinite for infinitely many $n$ (actually on a set in $\mathcal{U}$ ). Define a sequence of finite sets $\left\langle s_{i}: i \in \omega\right\rangle$ such that $\left(s_{i+1} \backslash s_{i}\right) \cap X_{j} \neq \emptyset$ for each $j \leq i$ such that $X_{j}$ is infinite. Clearly

$$
(\forall Y \in \mathcal{F})\left(\forall^{\infty} i\right) Y \cap X \cap s_{i} \neq \emptyset
$$

and thus $\langle\mathcal{F}, X\rangle$ is meager.

Claim 2. $\mathcal{F}$ is not weakly hereditarily meager.

Define $f \in{ }^{\omega} \omega$ by $f(n, m)=n$, the downward projection. Then no $f^{-1}(i)$ is compatible with $\mathcal{F}$ but $f(\mathcal{F})=\mathcal{U}$ is not meager.

EXAMPLE 3.3 (A completely meager $\mathrm{P}^{+}$-filter which is not a strong $\mathrm{P}^{+}$filter). We build a mad family $\mathcal{A}$ such that the filter $\mathcal{F}(\mathcal{A})$ generated by the complements of members of $\mathcal{A}$ has the required properties. It is essentially proved in [3] that any filter of the form $\mathcal{F}(\mathcal{A})$ will necessarily be a completely meager $\mathrm{P}^{+}$-filter; we include the proof for completeness.

CLAIM 1. All filters of the form $\mathcal{F}(\mathcal{A})$ are completely meager $\mathrm{P}^{+}$-filters.

Notice that a set $X \in \mathcal{F}(\mathcal{A})^{+}$if and only if $X$ has infinite intersection with infinitely many members of $\mathcal{A}$. Now if $Y_{0} \supseteq Y_{1} \supseteq \ldots$ are from $\mathcal{F}(\mathcal{A})^{+}$, choose $\left\{A_{n}: n \in \omega\right\} \subseteq \mathcal{A}$ such $Y_{n} \cap A_{m}$ is infinite for each $n, m$ as follows. Having obtained $\left\langle A_{0}, \ldots, A_{m-1}\right\rangle$, choose an infinite set $X \subseteq^{*} Y_{k} \backslash\left(A_{0} \cup \ldots\right.$ $\left.\ldots \cup A_{m-1}\right)$ for each $k$. Now since $\mathcal{A}$ is a mad family, there must be an $A_{m} \in \mathcal{A}$ with infinite intersection with $X$.

Finally, define a sequence $\left\langle n_{k}: k \in \omega\right\rangle$ such that

$$
Y_{k} \cap A_{l} \cap\left[n_{k}, n_{k+1}\right) \neq \emptyset
$$


for each $k$ and $l \leq k$. Clearly $Y=\bigcup_{k} Y_{k} \cap\left[n_{k}, n_{k+1}\right) \in \mathcal{F}(\mathcal{A})^{+}$and is almost included in each $Y_{n}$. Actually, we see that for any $X \in \mathcal{F}(\mathcal{A}), X \cap Y \cap$ $\left[n_{k}, n_{k+1}\right) \neq \emptyset$ for all but finitely many $k$ and thus we have also proved that $\mathcal{F}(\mathcal{A})$ is completely meager.

Now to make sure that $\mathcal{F}(\mathcal{A})$ is not a strong $\mathrm{P}^{+}$-filter, first fix an almost disjoint family $\mathcal{B}=\left\{B_{\alpha}: \alpha<\mathfrak{c}\right\}$ of size continuum and fix an enumeration of $\omega_{\omega}=\left\{f_{\alpha}: \alpha<\mathfrak{c}\right\}$. Fix further a partition $\left\langle X_{n}: n \in \omega\right\rangle$ of $\omega$. Now we define an almost disjoint family

$$
\mathcal{A}^{\prime}=\left\{\bigcup_{n \in B_{\alpha}} X_{n} \cap f_{\alpha}(n): \alpha<\mathfrak{c}\right\} .
$$

Let $\mathcal{A}$ be any mad family extending $\mathcal{A}^{\prime}$ together with a partition of the $X_{n}$ 's into countably many infinite sets (to make sure that $X_{n} \in \mathcal{F}(\mathcal{A})^{+}$). Clearly $\mathcal{F}(\mathcal{A})$ is not a strong $\mathrm{P}^{+}$-filter.

EXAMPle 3.4 (A filter with the strong Baire property which is not a $\mathrm{P}^{+}$-filter). Let $\mathcal{F}=\operatorname{Fr} \otimes F r$. Then $\mathcal{F}$ is a Borel filter and thus has the strong Baire property. If we define $Y_{n}=\{(l, m): l \geq n\} \in \mathcal{F}$, then $\mathcal{F}$ contains the complement of all $Y$ almost included in each $Y_{n}$.

EXAMPLE 3.5 (A non-meager $\mathrm{P}^{+}$-filter). It is worth noticing that the concept of $\mathrm{P}^{+}$-filter and the traditional notion of $\mathrm{P}$-filter are incomparable, that is, one does not imply the other. The difficult question of producing a non-meager P-filter has been around for some time; see [2] for some results and applications in this area.

We show that Simon's example [5] of a non-meager filter generated by $\mathfrak{b}$ sets is actually a $\mathrm{P}^{+}$-filter. As in his construction, we consider two possibilities.

Case $1: \mathfrak{b}<\mathfrak{d}$. Choose an unbounded family $\left\langle f_{\alpha}: \alpha<\mathfrak{b}\right\rangle$ of strictly increasing functions such that

$$
\alpha<\beta \rightarrow f_{\alpha}<^{*} f_{\beta} .
$$

Fix now $g \in{ }^{\omega} \omega$ not dominated by any $f_{\alpha}$, put

$$
X_{\alpha}=\left\{n: f_{\alpha}(n)<g(n)\right\}
$$

and define $\mathcal{F}=\left\langle X_{\alpha}: \alpha<\mathfrak{b}\right\rangle$. Then $\mathcal{F}$ is a P-filter and the next lemma shows that it really is a $\mathrm{P}^{+}$-filter.

LEMma 3.6. Any filter generated by less than $\mathfrak{d}$ sets is a $\mathrm{P}^{+}$-filter.

Let $\mathcal{H}$ be a filter generated by $\left\langle X_{\alpha}: \alpha<\lambda\right\rangle$ for some $\lambda<\mathfrak{d}$, and fix a descending sequence $Y_{0} \supseteq Y_{1} \supseteq \ldots$ from $\mathcal{H}^{+}$. For each $\alpha<\lambda$, choose $h_{\alpha} \in{ }^{\omega} \omega$ such that

$$
(\forall n) Y_{n} \cap X_{\alpha} \cap\left[n, h_{\alpha}(n)\right) \neq \emptyset \text {. }
$$


Since $\lambda<\mathfrak{d}$, fix $h \in{ }^{\omega} \omega$ not dominated by any $h_{\alpha}$ and let $Y=\bigcup_{n} Y_{n} \cap h(n)$ almost included in each $Y_{n}$. But for each $\alpha$, if $h_{\alpha}(n) \leq h(n), Y \cap X_{\alpha} \cap$ $[n, h(n)) \neq \emptyset$ and therefore $Y \in \mathcal{H}^{+}$.

Finally, we must show that $\mathcal{F}$ is non-meager. So suppose we are given a sequence $n_{0}<n_{1}<\ldots$ such that

$$
(\forall X \in \mathcal{F})\left(\forall^{\infty} k\right) X \cap\left[n_{k}, n_{k+1}\right) \neq \emptyset .
$$

Then for each $\alpha$, choose $m$ such that $X_{\alpha} \cap\left[n_{k}, n_{k+1}\right) \neq \emptyset$ for each $k \geq m$. Therefore for each $l, f_{\alpha}(l) \leq n_{l+m+1}$ and thus for all $l \geq m+1$ we have $f_{\alpha}(l) \leq n_{2 l}$. We conclude that the family $\left\langle f_{\alpha}: \alpha<\mathfrak{b}\right\rangle$ is bounded by $f(l)=n_{2 l}$, a contradiction.

Case $2: \mathfrak{b}=\mathfrak{d}$. Choose a scale $\left\langle f_{\alpha}: \alpha<\mathfrak{b}=\mathfrak{d}\right\rangle$ such that

1. Each $f_{\alpha}$ is strictly increasing and positive.

2. $(\forall \beta<\alpha)\left(\forall^{\infty} n\right)(\exists l) f_{\alpha}(n)<f_{\beta}(l)<f_{\beta}(l+1)<f_{\alpha}(n+1)$.

For each $\alpha$, put $X_{\alpha}=\bigcup_{n}\left[f_{\alpha}^{2 n}(0), f_{\alpha}^{2 n+1}(0)\right)$ and define $\mathcal{F}=\left\langle X_{\alpha}: \alpha<\right.$ $\mathfrak{b}=\mathfrak{d}\rangle$. We must show that $\mathcal{F}$ is a non-meager $\mathrm{P}^{+}$-filter.

Lemma 3.7. $\left(\forall A \in[\omega]^{\omega}\right)(\exists \alpha)\left(\forall^{\infty} n\right) A \cap\left[f_{\alpha}^{n}(0), f_{\alpha}^{n+1}(0)\right) \neq \emptyset$.

Proof. Given $A$, define $f \in{ }^{\omega} \omega$ such that $A \cap[n, f(n)) \neq \emptyset$ for each $n$. Now there must be an $\alpha$ such that $f \leq^{*} f_{\alpha}$ and therefore

$$
\left(\forall^{\infty} n\right) A \cap\left[n, f_{\alpha}(n)\right) \neq \emptyset .
$$

Thus $\left(\forall^{\infty} n\right) A \cap\left[f_{\alpha}(n), f_{\alpha}\left(f_{\alpha}(n)\right)\right) \neq \emptyset$, and finally

$$
\left(\forall^{\infty} n\right) A \cap\left[f_{\alpha}^{n}(0), f_{\alpha}^{n+1}(0)\right) \neq \emptyset
$$

as desired.

We are ready to show that $\mathcal{F}$ is a $\mathrm{P}^{+}$-filter. Fix a descending sequence $Y_{0} \supseteq Y_{1} \supseteq \ldots$ from $\mathcal{F}^{+}$. By induction, define a sequence of ordinals $\alpha_{n}<$ $\mathfrak{b}=\mathfrak{d}$ by $\alpha_{0}=0$ and given $\alpha_{n}$, let $\mathcal{F}_{n}$ be the filter generated by

$$
\left\{X_{\gamma}: \gamma<\alpha_{n}\right\} \cup\left\{Y_{k}: k \in \omega\right\} .
$$

Now choose $\alpha_{n+1}>\alpha_{n}$ so that

$$
\left(\forall Y \in \mathcal{F}_{n}\right)\left(\forall^{\infty} k\right) Y \cap\left[f_{\alpha_{n+1}}^{k}(0), f_{\alpha_{n+1}}^{k+1}(0)\right) \neq \emptyset .
$$

This is possible using the lemma and the fact that $\mathcal{F}_{n}$ is generated by less than $\mathfrak{b}=\mathfrak{d}$ sets. Finally, let $\alpha=\sup \left\{\alpha_{n}: n \in \omega\right\}$. We thus get

$$
\left(\forall Y \in \mathcal{F}_{\alpha}:=\left\langle X_{\gamma}: \gamma\langle\alpha\rangle \cup\left\langle Y_{n}: n \in \omega\right\rangle\right)\left(\forall^{\infty} k\right) Y \cap\left[f_{\alpha}^{k}(0), f_{\alpha}^{k+1}(0)\right) \neq \emptyset .\right.
$$

Therefore it suffices to find $Z \subseteq^{*} Y_{n}$ such that

1. $Z \in \mathcal{F}_{\alpha}^{+}$.

2. $\left(\forall^{\infty} k\right) Z \cap\left[f_{\alpha}^{k}(0), f_{\alpha}^{k+1}(0)\right) \neq \emptyset$. 
But again, $\mathcal{F}_{\alpha}$ is generated by less than $\mathfrak{b}$ sets, say by $\left\langle X_{\beta}: \beta<\lambda\right\rangle$ for some $\lambda<\mathfrak{b}$, and we may assume that this collection is also closed under finite intersections. Now for each such $\beta$, choose $h_{\beta} \in{ }^{\omega} \omega$ such that $Z_{\beta}=\bigcup_{n} Y_{n} \cap h_{\beta}(n)$ satisfies:

1. $\left(\forall^{\infty} k\right) Z_{\beta} \cap\left[f_{\beta}^{k}(0), f_{\beta}^{k+1}(0)\right) \neq \emptyset$.

2. $Z_{\beta} \cap X_{\beta}$ is infinite.

Now choose $h^{*}>h_{\beta}$ for $\beta<\lambda$. Then $Z=\bigcup_{n} Y_{n} \cap h(n)$ is as desired.

ExAmPle $3.8(\mathrm{CH})$ (A strong $\mathrm{P}^{+}$-filter not included in any $\mathrm{F}_{\sigma}$-filter). Enumerate all closed sets which generate a proper filter as $\left\langle\mathcal{C}_{\alpha}: \alpha<\omega_{1}\right\rangle$ and all sequences of potential candidates for members of $\mathcal{F}^{+}$as $\left\langle X_{n}^{\alpha}: n \in \omega\right\rangle$ for $\alpha<\omega_{1}$. We build a filter $\mathcal{F}$ in $\omega_{1}$ stages. At stage $\alpha$, assume that we have for $\beta<\alpha$ :

1. A sequence $\left\langle a_{n}^{\beta}: n \in \omega\right\rangle$ of finite sets.

2. $X_{\beta} \in[\omega]^{\omega}$ such that $\lim _{n}\left|X_{\beta} \cap a_{n}^{\gamma}\right|=\infty$ for all $\gamma<\alpha$.

3. $\beta<\gamma<\alpha \rightarrow X_{\gamma} \subseteq{ }^{*} X_{\beta}$.

Let $\mathcal{F}_{\alpha}$ be the filter generated by $\left\{X^{\mathrm{c}}:(\forall n)\left|X \cap a_{n}^{\beta}\right| \leq m\right\}$ for $m \in \omega$ and by $\left\{X_{\beta}: \beta<\alpha\right\}$. We are given a closed set $\mathcal{C}=\mathcal{C}_{\alpha}$ and we want to make sure that $\mathcal{F}_{\alpha} \nsubseteq\langle\mathcal{C}\rangle$. If this is already the case then there is nothing more to do; otherwise we may as well assume that $\alpha=\omega$.

Choose a sequence $n_{0}<n_{1}<\ldots$ such that

$$
\left(\forall Y_{1}, \ldots, Y_{k} \in \mathcal{C}\right) Y_{1} \cap \ldots \cap Y_{k} \cap\left[n_{k}, n_{k+1}\right) \neq \emptyset .
$$

Claim. $(\forall \beta<\alpha)(\forall m)(\forall Z)$, if $\left|Z \cap a_{k}^{\beta}\right| \leq m$ for each $k$, then

$$
(\forall Y \in \mathcal{C})\left(\forall^{\infty} l\right)\left(Y \cap\left[n_{l}, n_{l+1}\right)\right) \backslash Z \neq \emptyset .
$$

Pro of. Indeed, we have made sure to put $Z^{\mathrm{c}}$ in $\mathcal{F}_{\alpha}$ for all such $Z$ 's, and $\mathcal{F}_{\alpha} \subseteq\langle\mathcal{C}\rangle$ by assumptions.

The construction of $X_{\alpha}$ can therefore be obtained by a dovetailed construction. Now to handle the sequence $\left\langle X_{n}^{\alpha}: n \in \omega\right\rangle$, given that each $X_{n}^{\alpha} \in\left\langle\mathcal{F}_{\alpha}, X_{\alpha}\right\rangle^{+}$, choose $f \in{ }^{\omega} \omega$ such that

$$
(\forall n)\left|X_{\alpha} \cap X_{n}^{\alpha} \cap[n, f(n))\right| \geq n,
$$

and define $a_{n}^{\alpha}=[n, f(n)) \cap X_{n}^{\alpha}$.

This completes the construction. $\mathcal{F}=\bigcup_{\alpha} \mathcal{F}_{\alpha}$ is the desired filter.

EXAMPLE $3.9(\mathrm{CH})\left(\mathrm{A}\right.$ mad family $\mathcal{A}$ such that $\mathcal{F}(\mathcal{A})$ is a strong $\mathrm{P}^{+}$filter). In the following, $\mathcal{I}(\mathcal{A})$ denotes the ideal generated by members of $\mathcal{A}$. We start with a lemma.

Lemma $3.10(\mathrm{CH})$. There is a mad family $\mathcal{A}$ such that

$(\forall X \notin \mathcal{F}(\mathcal{A}) \cup \mathcal{I}(\mathcal{A}))(\exists \alpha)(\forall \beta>\alpha) A_{\beta} \backslash X$ and $A_{\beta} \cap X$ are infinite. 
Proof. Start with a partition $\left\langle A_{n}: n \in \omega\right\rangle$ of $\omega$ into infinite sets. Now enumerate $[\omega]^{\omega}$ as $\left\langle X_{\alpha}: \alpha<\omega_{1}\right\rangle$. Suppose that at stage $\alpha<\omega_{1}$, we have $\mathcal{A}_{\alpha}=\left\langle A_{\beta}: \beta\langle\alpha\rangle\right.$ and $\mathcal{X}_{\alpha} \subseteq[\omega]^{\omega} \backslash\left[\mathcal{F}\left(\mathcal{A}_{\alpha}\right) \cup \mathcal{I}\left(\mathcal{A}_{\alpha}\right)\right]$. Relist $\mathcal{A}_{\alpha}$ as $\left\langle B_{n}: n \in \omega\right\rangle$.

Case 1: $X_{\alpha} \in \mathcal{F}\left(\mathcal{A}_{\alpha}\right) \cup \mathcal{I}\left(\mathcal{A}_{\alpha}\right)$. There is nothing to do.

Case 2: $X_{\alpha} \notin \mathcal{F}\left(\mathcal{A}_{\alpha}\right) \cup \mathcal{I}\left(\mathcal{A}_{\alpha}\right)$. Thus in particular both $X_{\alpha} \nsubseteq^{*} B_{0} \cup \ldots$ $\ldots \cup B_{n}$ and $X_{\alpha}^{\mathrm{c}} \nsubseteq^{*} B_{0} \cup \ldots \cup B_{n}$ for each $n$.

List $\mathcal{X}_{\alpha} \cup\left\{X_{\alpha}\right\}$ as $\left\langle X_{n}: n \in \omega\right\rangle$ and build $A_{\alpha}$ such that:

1. $(\forall n) A_{\alpha} \cap B_{n}={ }^{*} \emptyset$.

2. $(\forall n) A_{\alpha} \backslash X_{n}$ and $A_{\alpha} \cap X_{n}$ are infinite.

At stage $n$, assume that $a_{n} \in[\omega]^{<\omega}$, then choose

$$
\begin{array}{ll}
k_{i} \in X_{i} \backslash\left(B_{0} \cup \ldots \cup B_{n} \cup n\right) & \text { for } i<n, \\
l_{i} \in X_{i}^{\mathrm{c}} \backslash\left(B_{0} \cup \ldots \cup B_{n} \cup n\right) & \text { for } i<n,
\end{array}
$$

and let $a_{n+1}=a_{n} \cup\left\{k_{i}, l_{i}\right\}_{i<n}$. Hence $A_{\alpha}=\bigcup_{n} a_{n}$ satisfies $1-2$ and put $\mathcal{A}_{\alpha+1}=\mathcal{A}_{\alpha} \cup\left\{A_{\alpha}\right\}, \mathcal{X}_{\alpha+1}=\mathcal{X}_{\alpha} \cup\left\{X_{\alpha}\right\} \subseteq[\omega]^{\omega} \backslash\left[\mathcal{F}\left(\mathcal{A}_{\alpha+1}\right) \cup \mathcal{I}\left(\mathcal{A}_{\alpha+1}\right)\right]$. This completes the construction. The verification that $\mathcal{A}=\left\{A_{\alpha}: \alpha<\omega_{1}\right\}$ has the desired properties is straightforward.

Now we show that such a mad family yields a strong $\mathrm{P}^{+}$-filter $\mathcal{F}(\mathcal{A})$. But given a sequence $\left\langle X_{n}: n \in \omega\right\rangle$ from $\mathcal{F}(\mathcal{A})^{+}$, we can choose by the assumptions on $\mathcal{A}$ countably many members $\left\langle A_{n}: n \in \omega\right\rangle$ from $\mathcal{A}$ such that $A_{n} \cap X_{m}$ is infinite for each $n$ and $m$. Now define $f \in{ }^{\omega} \omega$ such that

$$
(\forall n)(\forall i<n) A_{i} \cap X_{n} \cap[n, f(n)) \neq \emptyset .
$$

If $Y \in \mathcal{F}(\mathcal{A})$, there must be an $A_{n}$ such that $A_{n} \subseteq^{*} Y$ and therefore $Y \cap X_{n} \cap f(n) \neq \emptyset$ for all but finitely many $n$.

4. Conclusion. The two implications that remain to be solved are the following.

Question 1. Does a completely meager $\mathrm{P}^{+}$-filter, or actually even a hereditarily meager filter necessarily have the strong Baire property?

One remains in ZFC.

Question 2. Is there a strong $\mathrm{P}^{+}$-filter not included in any $\mathrm{F}_{\sigma}$-filter?

We still have very little understanding of the structure of filters of the form $\mathcal{F}(\mathcal{A})$ for a mad family $\mathcal{A}$. In particular, the following remains unsolved.

Question 3. Do all filters of the form $\mathcal{F}(\mathcal{A})$ for a mad family $\mathcal{A}$ have the strong Baire property? 
We have, however, a meagerness property that separates filters of the form $\mathcal{F}(\mathcal{A})$ and $\mathrm{F}_{\sigma}$-filters, namely the strong $\mathrm{P}^{+}$property. In view of the results of [3] that $\mathrm{F}_{\sigma}$-filters can be diagonalized in a forcing extension without adding an unbounded real, we may ask the following.

Question 4. Can all strong $\mathrm{P}^{+}$-filters be diagonalized in a forcing extension without adding unbounded reals?

Of course the fundamental motivation for these questions is:

Question 5. Can filters of the form $\mathcal{F}(\mathcal{A})$ for a mad family $\mathcal{A}$ be diagonalized in a forcing extension without adding an unbounded real?

\section{References}

[1] T. Bartoszyński and H. Judah, Measure and category - filters on $\omega$, in: Set Theory of the Continuum, H. Judah, W. Just and H. Woodin (eds.), Springer, 1992, 175-201.

[2] W. Just, A. R. D. Mathias, K. Prikry and P. Simon, On the existence of large P-ideals, J. Symbolic Logic 55 (1990), 457-465.

[3] C. Laflamme, Zapping small filters, Proc. Amer. Math. Soc. 114 (1992), 535-544.

[4] S. Shelah, Cardinal invariants of the continuum, in: Axiomatic Set Theory, J. Baumgartner, D. Martin and S. Shelah (eds.), Contemp. Math. 31, Amer. Math. Soc., 1984, 183-207.

[5] P. Simon, private communication, August 1987.

[6] M. Talagrand, Compacts de fonctions mesurables et filtres non mesurables, Studia Math. 67 (1980), 13-43.

[7] —, Filtres: mesurabilité, rapidité, propriété de Baire forte, ibid. 74 (1982), 283-291.

DEPARTMENT OF MATHEMATICS AND STATISTICS

UNIVERSITY OF CALGARY

CALGARY, ALBERTA

CANADA T2N $1 \mathrm{~N} 4$

Received 7 December 1993;

in revised form 7 November 1994 